\title{
Enumeration of Varlet and Comer hypergroups
}

\author{
H. Aghabozorgi \\ Department of Mathematics \\ Yazd University, Yazd, Iran \\ h_aghabozorgi1@yahoo.com \\ M. Jafarpour \\ Department of Mathematics \\ Vali-e-Asr University of Rafsanjan, Rafsanjan, Iran \\ m.j@mail.vru.ac.ir \\ B. Davvaz \\ Department of Mathematics \\ Yazd University, Yazd, Iran \\ davvaz@yazduni.ac.ir
}

Submitted: Feb 12, 2011; Accepted: May 29, 2011; Published: Jun 14, 2011

Mathematics Subject Classifications: 20N20, 05E15.

\begin{abstract}
In this paper, we study hypergroups determined by lattices introduced by Varlet and Comer, especially we enumerate Varlet and Comer hypergroups of orders less than 50 and 13, respectively.
\end{abstract}

\section{Basic definitions and results}

An algebraic hyperstructure is a natural generalization of a classical algebraic structure. More precisely, an algebraic hyperstructure is a non-empty set $H$ endowed with one or more hyperoperations that associate with two elements of $H$ not an element, as in a classical structure, but a subset of $H$. One of the interests of the researchers in the field of hyperstructures is to construct new hyperoperations using graphs [18], binary relations $[2,5,7,8,9,11,15,21,23], n$-ary relations [10], lattices [16], classical structures [13], tolerance space [12] and so on. Connections between lattices and hypergroupoids have been considered since at least three decades, starting with [24] and followed by [3, 14, 17]. This paper deals with hypergroups derived from lattices, in particular we study some properties of the hypergroups defined by J.C. Varlet [24] and S. Comer [3] that called here Varlet hypergroups and Comer hypergroups, respectively. Using the results of $[1,22]$ 
we enumerate the number of non isomorphic Varlet and Comer hypergroups of orders less than 50 and 13, respectively.

Let us briefly recall some basic notions and results about hypergroups; for a comprehensive overview of this subject, the reader is referred to [4, 6, 25]. For a non-empty set $H$, we denote by $\mathcal{P}^{*}(H)$ the set of all non-empty subsets of $H$. A non-empty set $H$, endowed with a mapping, called hyperoperation, o: $H^{2} \longrightarrow \mathcal{P}^{*}(H)$ is named hypergroupoid. A hypergroupoid which satisfies the following conditions: (1) $(x \circ y) \circ z=x \circ(y \circ z)$, for all $x, y, z \in H$ (the associativity), (2) $x \circ H=H=H \circ x$, for all $x \in H$ (the reproduction axiom) is called a hypergroup. In particular, an associative hypergroupoid is called a semihypergroup and a hypergroupoid that satisfies the reproduction axiom is called a quasihypergroup. If $A$ and $B$ are non-empty subsets of $H$, then $A \circ B=\bigcup_{a \in A, b \in B} a \circ b$. Let $(H, \circ)$ and $\left(H^{\prime}, o^{\prime}\right)$ be two hypergroups. A function $f: H \longrightarrow H^{\prime}$ is called a homomorphism if it satisfies the condition: for any $x, y \in H, f(x \circ y) \subseteq f(x) \circ^{\prime} f(y)$. $f$ is a good homomorphism if, for any $x, y \in H, f(x \circ y)=f(x) \circ^{\prime} f(y)$. We say that the two hypergroups are isomorphic if there is a good homomorphism between them which is also a bijection.

Join spaces were introduced by W. Prenowitz and then applied by him and J. Jantosciak both in Euclidian and in non Euclidian geometry [19, 20]. Using this notion, several branches of non Euclidian geometry were rebuilt: descriptive geometry, projective geometry and spherical geometry. Then, several important examples of join spaces have been constructed in connection with binary relations, graphs and lattices. In order to define a join space, we need the following notation: If $a, b$ are elements of a hypergroupoid $(H, \circ)$, then we denote $a / b=\{x \in H \mid a \in x \circ b\}$. Moreover, by $A / B$ we intend the set $\bigcup_{a \in A, b \in B} a / b$.

A commutative hypergroup $(H, \circ)$ is called a join space if the following condition holds for all elements $a, b, c, d$ of $H$ :

$$
a / b \cap c / d \neq \emptyset \Longrightarrow a \circ d \cap b \circ c \neq \emptyset \text {. }
$$

Definition 1.1. [24] Let $\mathfrak{L}_{\leq}=(L, \wedge, \vee)$ be a lattice with join $\vee$, meet $\wedge$ and order relation $\leq$ and let:

$$
\forall(a, b) \in L^{2}, \quad a \circ b=\{x \in L \quad \mid a \wedge b \leq x \leq a \vee b\}
$$

Theorem 1.2. [24] For a lattice $\mathfrak{L}_{\leq}$the following are equivalent:

(1) $\mathfrak{L}_{\leq}$is distributive;

(2) $\mathbb{L}_{\leq}=(L, \circ)$ is a join space.

The class of intervals of elements of $\mathfrak{L}_{\leq}=(L, \wedge, \vee)$ is denoted by $I\left(\mathfrak{L}_{\leq}\right)$, that is:

$$
I\left(\mathfrak{L}_{\leq}\right)=\left\{[a, b] \mid(a, b) \in L^{2}, a \leq b\right\},
$$

where $[a, b]=\{x \in L \mid a \leq x \leq b\}$. 
Theorem 1.3. For the join space $\mathbb{L}_{\leq}$given in Theorem 1.2, the following equality holds:

$$
\operatorname{Sub}\left(\mathbb{L}_{\leq}\right)=I\left(\mathfrak{L}_{\leq}\right)=\left\{x \circ y \mid(x, y) \in L^{2}\right\},
$$

where $\operatorname{Sub}\left(\mathbb{L}_{\leq}\right)$is the class of subhypergroups of $\mathbb{L}_{\leq}$.

Proof. Let $[a, b] \in I\left(\mathfrak{L}_{\leq}\right)$. Then, for any $x, y \in[a, b]$ we have $a \leq x \leq b$ and $a \leq y \leq b$. These lead to $a \leq x \wedge y \leq x \vee y \leq b$ and so $x \circ y=[x \wedge y, x \vee y] \subseteq[a, b]$. Moreover, $[a, b] \circ x=x \circ[a, b]=\bigcup_{t \in[a, b]} x \circ t=[a, x] \cup[x, b]=[a, b]$. Conversely, let $H \in S u b\left(\mathbb{L}_{\leq}\right)$, $a \circ b \subseteq H$, for all $a, b \in H$. Hence, $[a \wedge b, a \vee b] \subseteq H$. In particular, one obtains that $H$ is closed under the operations $\wedge$ and $\vee$. Let $A=\left\{a_{i}\right\}_{i \in I}$ and $B=\left\{b_{i}\right\}_{i \in J}$ are the sets of minimal and maximal elements of $H$, respectively with respect to the order on $L$. If $|I| \geq 2$, then we can choose two distinct elements of A, say $a, a^{\prime}$, it follows that $a \wedge a^{\prime} \in H$ a contradiction. In this way, $A$ contains a unique element, say $a_{0}$. Similarly, $B$ contains a unique element, say $b_{0}$. It is clear that $H=\left[a_{0}, b_{0}\right]$. We can easily see that the equality $I\left(\mathfrak{L}_{\leq}\right)=\left\{x \circ y \mid(x, y) \in L^{2}\right\}$ holds.

Theorem 1.4. Let $\mathfrak{L}_{\leq}=(L, \wedge, \vee)$ be a distributive lattice. Define on the set $I\left(\mathfrak{L}_{\leq}\right)$, the following hyperoperation

$$
[x, y] \odot_{R}[z, w]=S u b([x \wedge z, y \vee w]) .
$$

Then, $\left(I\left(\mathfrak{L}_{\leq}\right), \odot_{R}\right)$ is a hypergroup.

Proof. Using previous theorem, it is clear that $\odot_{R}$ is a well defined hyperoperation. We prove $\odot_{R}$ is associative. To this end we have:

$$
\begin{aligned}
\left(\left[x_{1}, y_{1}\right] \odot_{R}\left[x_{2}, y_{2}\right]\right) \odot_{R}\left[x_{3}, y_{3}\right] & =\operatorname{Sub}\left(\left[x_{1} \wedge x_{2}, y_{1} \vee y_{2}\right]\right) \odot_{R}\left[x_{3}, y_{3}\right] \\
& =\operatorname{Sub}\left(\left[\left(x_{1} \wedge x_{2}\right) \wedge x_{3},\left(y_{1} \vee y_{2}\right) \vee y_{3}\right]\right) \\
& =\operatorname{Sub}\left(\left[x_{1} \wedge\left(x_{2} \wedge x_{3}\right), y_{1} \vee\left(y_{2} \vee y_{3}\right)\right]\right) \\
& =\left[x_{1}, y_{1}\right] \odot_{R} \operatorname{Sub}\left(\left[x_{2} \wedge x_{3}, y_{2} \vee y_{3}\right]\right) \\
& =\left[x_{1}, y_{1}\right] \odot_{R}\left(\left[x_{2}, y_{2}\right] \odot_{R}\left[x_{3}, y_{3}\right]\right) .
\end{aligned}
$$

\section{Enumeration of finite Varlet hypergroups}

It is well known that every binary relation $\rho$ on a finite set $L$, with $\operatorname{car} d L=n$, may be represented by a Boolean matrix $M(\rho)$ and conversely every Boolean matrix of order $n$ defines on $L$ a binary relation. Indeed, let $L=\left\{a_{1}, \ldots, a_{n}\right\}$; a Boolean matrix of order $n$ is constructed in the following way: the element in the position $(i, j)$ of the matrix is 1 , if $\left(a_{i}, a_{j}\right) \in \rho$ and it is 0 if $\left(a_{i}, a_{j}\right) \notin \rho$ and vice versa. Hence, on every set with $n$ elements, $2^{n^{2}}$ partial hypergroupoids can be defined. Recall that in a Boolean algebra the following properties hold: $0+1=1+0=1+1=1$, while $0+0=0$, and $0 \cdot 0=0 \cdot 1=1 \cdot 0=0$, $1 \cdot 1=1$. Moreover, if $\rho$ is a binary relation on $L$, then $M\left(\rho^{2}\right)=M^{2}(\rho)$. 
Proposition 2.1. Let $\mathfrak{L}_{\leq}$and $\mathfrak{L}_{<^{\prime}}$ be two finite lattices and $\left(t_{i j}\right),\left(t_{i j}^{\prime}\right)$ be their associated matrices, respectively. Then, $\mathfrak{L}_{\leq}$and $\mathfrak{L}_{\leq^{\prime}}$ are isomorphic if and only if $t_{i j}=t_{\sigma(i) \sigma(j)}^{\prime}$, for a permutation $\sigma$ of the set $\{1,2, \ldots, n\}$.

Definition 2.2. Let $\mathfrak{L}_{\leq}$be a finite lattice. The matrix $M(\leq)$ is called very good if and only if $\mathbb{L}_{\leq}$is a Varlet hypergroup.

Proposition 2.3. If $M=\left(t_{i j}\right)_{n \times n}$ is a very good matrix and $M^{2}=\left(s_{i j}\right)$, then the following assertions hold:

(1) $t_{i i}=1$, for all $1 \leq i \leq n$;

(2) $t_{i j}=1 \Rightarrow t_{j i}=0$, for all $i \neq j$ and $1 \leq i, j \leq n$;

(3) $M^{2} \leq M$, (i.e., $s_{i j}=1 \Rightarrow t_{i j}=1$, for all $\left.1 \leq i, j \leq n\right)$;

(4) there exists $i$, with $1 \leq i \leq n$, such that $t_{i j}=1$, for all $1 \leq j \leq n$;

(5) there exists $j$, with $1 \leq j \leq n$, such that $t_{i j}=1$, for all $1 \leq i \leq n$.

The matrix $T=\left(t_{i j}\right)_{n \times n}$, with

$$
t_{i j}= \begin{cases}1 & \text { if } i \leq j \\ 0 & \text { otherwise }\end{cases}
$$

for any $i, j \in\{1,2, \ldots, n\}$, is a very good matrix that we call it $n$-triangular and the corresponding hypergroup is a Varlet hypergroup.

In the following, we give in terms of matrices a necessary and sufficient condition such that two Varlet hypergroups associated with two lattices on the same set $L$, are isomorphic.

Proposition 2.4. Let $L=\left\{a_{1}, \ldots, a_{n}\right\}$ be a finite set, $\leq$ and $\leq \leq$ be two order relations on $L$ and $M(\leq)=\left(t_{i j}\right), M\left(\leq^{\prime}\right)=\left(t_{i j}^{\prime}\right)$ be their associated matrices. If $t_{i j}=t_{\sigma(i) \sigma(j)}^{\prime}$, for a permutation $\sigma$ of the set $\{1,2, \ldots, n\}$, then the following assertions hold:

(1) $a_{i} \leq a_{j} \Leftrightarrow a_{\sigma(i)} \leq^{\prime} a_{\sigma(j)}$;

(2) $a_{i} \wedge a_{j}=a_{k} \Leftrightarrow a_{\sigma(i)} \wedge a_{\sigma(j)}=a_{\sigma(k)}$;

(3) $a_{i} \vee a_{j}=a_{k} \Leftrightarrow a_{\sigma(i)} \vee a_{\sigma(j)}=a_{\sigma(k)}$.

Theorem 2.5. Let $\mathfrak{L}_{\leq}$and $\mathfrak{L}_{\leq}$be two finite distributive lattices and let $M(\leq)=\left(t_{i j}\right)$ and $M\left(\leq^{\prime}\right)=\left(t_{i j}^{\prime}\right)$ be their associated matrices. The hypergroups $\mathbb{L}_{\leq}$and $\mathbb{L}_{\leq^{\prime}}$ are isomorphic if and only if $t_{i j}=t_{\sigma(i) \sigma(j)}^{\prime}$, for a permutation $\sigma$ of the set $\{1,2, \ldots, n\}$. 
Proof. Let $L=\left\{a_{1}, \ldots, a_{n}\right\}$ and $\theta: \mathbb{L}_{\leq} \longrightarrow \mathbb{L}_{\leq^{\prime}}$ be an isomorphism. Then, $\theta\left(a_{i} \circ a_{j}\right)=$ $\theta\left(a_{i}\right) \circ^{\prime} \theta\left(a_{j}\right)$ and so

$$
\left\{\theta\left(a_{k}\right) \mid a_{i} \wedge a_{j} \leq a_{k} \leq a_{i} \vee a_{j}\right\}=\left\{a_{s} \mid \theta\left(a_{i}\right) \wedge \theta\left(a_{j}\right) \leq^{\prime} a_{s} \leq^{\prime} \theta\left(a_{i}\right) \vee \theta\left(a_{j}\right)\right\}
$$

Thus, we have $a_{i} \wedge a_{j} \leq a_{k} \leq a_{i} \vee a_{j}$ if and only if $\theta\left(a_{i}\right) \wedge \theta\left(a_{j}\right) \leq^{\prime} \theta\left(a_{k}\right) \leq^{\prime} \theta\left(a_{i}\right) \vee \theta\left(a_{j}\right)$. Suppose that $\theta\left(a_{j}\right)=a_{\sigma(j)}$, for a permutation $\sigma$ of the set $\{1,2, \ldots, n\}$. We show that $t_{i j}=t_{\sigma(i) \sigma(j)}^{\prime}$. If $t_{i j}=0$, then we can easily see that $t_{\sigma(i) \sigma(j)}^{\prime}=0$. Now, suppose that $t_{i j}=1$. Then, we have $t_{\sigma(i) \sigma(j)}^{\prime}=1$ or $t_{\sigma(j) \sigma(i)}^{\prime}=1$. Since $t_{j i}=0$ the case $t_{\sigma(j) \sigma(i)}^{\prime}=1$ would not occur. Thus, we have $t_{\sigma(i) \sigma(j)}^{\prime}=1$. Conversely, note that, for a permutation $\sigma$ of the set $\{1,2, \ldots, n\}$, we have $a_{i} \leq a_{j} \Leftrightarrow a_{\sigma(i)} \leq^{\prime} a_{\sigma(j)}$. Consider the map $\varphi: \mathbb{L}_{\leq} \rightarrow \mathbb{L}_{\leq^{\prime}}$ with $\varphi\left(a_{i}\right)=a_{\sigma(i)}$. Clearly, $\varphi$ is a bijection and by using previous proposition we have:

$$
\left.\left\{\varphi\left(a_{k}\right) \mid a_{i} \wedge a_{j} \leq a_{k} \leq a_{i} \vee a_{j}\right\}=\left\{a_{\sigma(k)} \mid a_{\sigma(i)} \wedge a_{\sigma(j)}\right) \leq^{\prime} a_{\sigma(k)} \leq^{\prime} a_{\sigma(i)} \vee a_{\sigma(j)}\right\} .
$$

Therefore, $\varphi\left(a_{i} \circ a_{j}\right)=\varphi\left(a_{i}\right) \circ^{\prime} \varphi\left(a_{j}\right)$ and the proof is completed.

We say that a Boolean matrix is reflexive, antisymmetric or transitive if the associated binary relation is reflexive, antisymmetric or transitive, respectively.

We say that two very good matrices are isomorphic if the Varlet hypergroups obtained by them are isomorphic.

Theorem 2.6. Let $M=\left(t_{i j}\right)_{n \times n}$ and $M^{\prime}=\left(t_{i j}^{\prime}\right)_{m \times m}$ be two very good matrices. Then, $M \oplus M^{\prime}=\left(m_{i j}\right)_{k \times k}$, where $k=n+m$, and

$$
m_{i j}= \begin{cases}t_{i j} & \text { if } i \leq n, j \leq n \\ t_{i j}^{\prime} & \text { if } n<i, n<j \\ 1 & \text { if } i \leq n, j>n \\ 0 & \text { if } n<i, j \leq n\end{cases}
$$

is a very good matrix.

Proof. Since $M \oplus M^{\prime}=\left(\begin{array}{cc}M & O^{\prime} \\ O & M^{\prime}\end{array}\right)_{k \times k}$, where $O$ is an $m \times n$ matrix which all entries are zero (i.e., $O=(0)_{m \times n}$ ), and $O^{\prime}$ is an $n \times m$ matrix which all entries are one. We have $\left(M \oplus M^{\prime}\right)^{2}=M^{2} \oplus M^{\prime 2} \leq M \oplus M^{\prime}$ and so $M \oplus M^{\prime}$ is a transitive matrix. Obviously, $M \oplus M^{\prime}$ is reflexive and antisymmetric. Now, suppose that $L=\left\{a_{1}, \ldots, a_{n+m}\right\}$ and $\leq$ is the associated binary relation of $M \oplus M^{\prime}$. Then

$$
a_{i} \leq a_{j} \Leftrightarrow\left[t_{i j}=1 \text { or } t_{i j}^{\prime}=1, \text { and }(i \leq n, \quad j>n)\right]
$$

Hence, $a_{i} \wedge\left(a_{j} \vee a_{k}\right) \leq\left(a_{i} \wedge a_{j}\right) \vee\left(a_{i} \wedge a_{k}\right)$, for every $\left(a_{i}, a_{j}, a_{k}\right) \in L^{3}$. So, $(L, \leq)$ is a distributive lattice and $M \oplus M^{\prime}$ is very good.

Corollary 2.7. Let $V_{n}$ be the number of non isomorphic Varlet hypergroups of order $n$. Then, $V_{n+m} \geq V_{n} V_{m}$, for all $n, m \in \mathbb{N}$.

Using the results of [22] we can enumerate the number of Varlet hypergroups (up to isomorphism) with cardinality less than 50 which we summarize at the following table. 


\begin{tabular}{|c|c||c|c|}
\hline $\mathrm{n}=$ & Number of Varlet hypergroups & $\mathrm{n}=$ & Number of Varlet hypergroups \\
\hline \hline 1 & 1 & 26 & 711811 \\
2 & 1 & 27 & 1309475 \\
3 & 1 & 28 & 2413144 \\
4 & 2 & 29 & 4442221 \\
5 & 3 & 30 & 8186962 \\
6 & 5 & 31 & 15077454 \\
7 & 8 & 32 & 27789108 \\
8 & 15 & 33 & 51193086 \\
9 & 26 & 34 & 94357143 \\
10 & 47 & 35 & 173859936 \\
11 & 82 & 36 & 320462062 \\
12 & 151 & 37 & 590555664 \\
13 & 269 & 38 & 1088548290 \\
14 & 494 & 39 & 2006193418 \\
15 & 891 & 40 & 3697997558 \\
16 & 1639 & 41 & 6815841849 \\
17 & 2978 & 42 & 12563729268 \\
18 & 5483 & 43 & 23157428823 \\
19 & 10006 & 44 & 42686759863 \\
20 & 18428 & 45 & 78682454720 \\
21 & 33749 & 46 & 145038561665 \\
22 & 62162 & 47 & 267348052028 \\
23 & 114083 & 492815778109 \\
24 & 210189 & 908414736485 \\
25 & 386292 & \\
\hline
\end{tabular}

\section{On Comer hypergroups}

Proposition 3.1. [3] Let $\mathfrak{L}_{\leq}=(L, \wedge, \vee)$ be a Modular lattice. If for all $a, b \in L$ we define

$$
a \bullet b=\{z \in L \quad \mid z \vee a=a \vee b=b \vee z\},
$$

then $\mathbf{L}_{\leq}=(L, \bullet)$ is a hypergroup that we call it "Comer hypergroup".

Definition 3.2. Let $\mathfrak{L}_{\leq}$be a finite lattice. The matrix $M(\leq)$ is called good if and only if $\mathbf{L}_{\leq}$is a Comer hypergroup.

Theorem 3.3. Let $\mathfrak{L}_{\leq}$and $\mathfrak{L}_{\leq}$be two finite modular lattices and $M(\leq)=\left(t_{i j}\right), M\left(\leq^{\prime}\right)=$ $\left(t_{i j}^{\prime}\right)$ be their associated matrices. The hypergroups $\mathbf{L}_{\leq}$and $\mathbf{L}_{\leq}$are isomorphic if and only if $t_{i j}=t_{\sigma(i) \sigma(j)}^{\prime}$, for a permutation $\sigma$ of the set $\{1,2, \ldots, n\}$. 
Theorem 3.4. Let $M=\left(t_{i j}\right)_{n \times n}, M^{\prime}=\left(t_{i j}^{\prime}\right)_{m \times m}$ be two good matrices. Then, $M \boxplus M^{\prime}=$ $\left(m_{i j}\right)_{k \times k}$, where $k=n+m$ and

$$
m_{i j}= \begin{cases}t_{i j} & \text { if } i \leq n, j \leq n \\ t_{t j}^{\prime} & \text { if } n<i, n<j \\ 1 & \text { if }\left(\prod_{s=1}^{n} t_{i s}=1, j>n\right) \text { or }\left(i \leq n, \prod_{l=1}^{m} t_{l j}^{\prime}=1\right) \\ 0 & \text { others }\end{cases}
$$

is a good matrix.

Proof. We have $M \boxplus M^{\prime}=\left(\begin{array}{cc}M & O^{\prime} \\ O & M^{\prime}\end{array}\right)_{k \times k}$, where $O=(0)_{m \times n}$ and $O^{\prime}=\left(b_{i j}\right)_{n \times m}$, where

$$
b_{i j}=1 \Leftrightarrow\left[\prod_{s=1}^{n} t_{i s}=1 \text { or } \prod_{l=1}^{m} t_{l j}^{\prime}=1\right] .
$$

So, $\left(M \boxplus M^{\prime}\right)^{2}=M^{2} \boxplus M^{\prime 2} \leq M \boxplus M^{\prime}$ and so $M \boxplus M^{\prime}$ is a transitive matrix. Notice that in $M$ just exists one row and one column which all entries are 1 . Now, suppose that $L=L_{1}=\left\{a_{1}, \ldots a_{n}\right\} \cup\left\{a_{n+1}, \ldots a_{n+m}\right\}=L_{2}$ and $\leq_{1} \leq_{1}$ and $\leq_{2}$ are the associated binary relations of $M \boxplus M^{\prime}, M$ and $M^{\prime}$ on $L, L_{1}$ and $L_{2}$, respectively. Then, we have

$$
a_{i} \leq a_{j} \Leftrightarrow\left[a_{i} \leq_{1} a_{j} \text {, or } a_{i} \leq_{2} a_{j} \text {, or } a_{i}=\bigwedge_{s=1}^{n} a_{s} \text { and or } a_{j}=\bigvee_{s=n+1}^{n+m} a_{s}\right]
$$

Hence, $\left(a_{i} \wedge a_{j}\right) \vee\left(a_{i} \wedge a_{k}\right)=a_{i} \wedge\left(a_{j} \vee\left(a_{i} \wedge a_{k}\right)\right)$, for every $\left(a_{i}, a_{j}, a_{k}\right) \in L^{3}$, so $(L, \leq)$ is a modular lattice and $M \boxplus M^{\prime}$ is good.

Corollary 3.5. If $C_{n}$ is the number of non isomorphic Comer hypergroups of order $n$, then $C_{n+m} \geq C_{n} C_{m}$, for all $n, m \in \mathbb{N}$.

Proposition 3.6. For every $n \in \mathbb{N}, V_{n} \leq C_{n}$.

ExAmple 1. Let $T$ and $T^{\prime}$ be 2-triangular and 3-triangular matrixes. Then, $T \boxplus T^{\prime}$ is a good matrix which is not very good.

By using the results of [1] we can count the number of Comer hypergroups (up to isomorphism) with the cardinality less than 13 which we summarize at the following table.

\begin{tabular}{|c||c|c|c|c|c|c|c|c|c|c|c|c|}
\hline $\mathrm{n}=$ & 1 & 2 & 3 & 4 & 5 & 6 & 7 & 8 & 9 & 10 & 11 & 12 \\
\hline Comer hypergroups & 1 & 1 & 1 & 2 & 4 & 8 & 16 & 34 & 72 & 157 & 343 & 766 \\
\hline
\end{tabular}




\section{References}

[1] R. Belohlavek and V. Vychodil, Residuated Lattices of Size $\leq$ 12, Mathematics and Statistics, 27 (2010), 147-161.

[2] J. Chvalina, Commutative hypergroups in the sense of Marty and ordered sets, Proceedings of the Summer School on General Algebra and Ordered Sets, 1994, Olomouc, Czech Republic, pp. 19-30.

[3] S. Comer, Multi-valued algebras and their graphical representations, Math. Comp. Sci. Dep. the Citadel. Charleston, South Carolina, 29409, July 1986.

[4] P. Corsini, Prolegomena of Hypergroups Theory, Aviani Editore, 1993.

[5] P. Corsini and V. Leoreanu, Hypergroups and binary relations, Algebra Universalis, 43 (2000), 321-330.

[6] P. Corsini and V. Leoreanu, Applications of hyperstructure theory, in: Advances in Mathematics, Kluwer Academic Publishers, Dordrecht, 2003.

[7] I. Cristea and M. Stefanescu, Binary relations and reduced hypergroups, Discrete Math., 308 (2008), 3537-3544.

[8] I. Cristea, M. Stefanescu and C. Angheluta, About the fundamental relations defined on the hypergroupoids associated with binary relations, European J. Combin., 32 (2011), 72-81.

[9] B. Davvaz and V. Leoreanu-Fotea, Binary relations on ternary hypergroups, Communications in Algebra, 38(10) (2010), 3621-3636.

[10] B. Davvaz and T. Vougiouklis, n-Ary hypergroups, Iranian Journal of Science and Technology, Transaction A, 30 (A2) (2006), 165-174.

[11] B. Davvaz, W.A. Dudek and S. Mirvakili, Neutral elements, fundamental relations and n-ary hypersemigroups, International Journal of Algebra and Computation, 19(4) (2009), 567-583.

[12] S. Hoskova and J. Chvalina, Discrete transformation hypergroups and transformation hypergroups with phase tolerance space, Discrete Math., 308 (2008), 4133-4143.

[13] M. Jafarpour and S.Sh. Mousavi, A construction of hypersemigroups from a family of preordered semigroups, Scientiae Mathematicae Japonicae On-line, e-2009, 587-594.

[14] M. Konstantinidou and K. Serafimidis, Sur les P-supertreillis, in: T. Vougiouklis, (Ed.), New Frontiers in Hyperstructures and Rel. Algebras, Hadronic Press, Palm Harbor, USA, 1996, pp. 139-148.

[15] V. Leoreanu-Fotea and B. Davvaz, n-hypergroups and binary relations, European J. Combin., 29 (2008), 1207-1218.

[16] V. Leoreanu-Fotea and B. Davvaz, Join n-spaces and lattices, Journal of MultipleValued Logic and Soft Computing, 15 (2009), 421-432.

[17] V. Leoreanu-Fotea and I.G. Rosenberg, Hypergroupoids determined by lattices, European J. Combin., 31 (2010), 925-931. 
[18] J. Nieminen, Join space graphs, J. Geom., 33 (1988), 99-103.

[19] W. Prenowitz and J. Jantosciak, Geometries and join spaces, J. Reine Angew. Math., 257 (1972), 100-128.

[20] W. Prenowitz and J. Jantosciak, Join geometries, Springer-Verlag, UTM, 1979.

[21] I.G. Rosenberg, Hypergroups and join spaces determined by relations, Italian J. Pure Appl. Math., 4 (1998), 93-101.

[22] M. Erne, J. Heitzig and J. Reinhold, On the Number of Distributive Lattices, Electron. J. Comb., 9(1) (2002), \#R24, 23 pp.

[23] S. Spartalis, The hyperoperation relation and the Corsini's partial or not-partial hypergroupoids (A classification), Ital. J. Pure Appl. Math., 24 (2008), 97-112.

[24] J.C. Varlet, Remarks on distributive lattices, Bull. de l'Acad. Polonnaise des Sciences, Serie des Sciences Math., Astr. et Phys., 23 (1975), 1143-1147.

[25] T. Vougiouklis, Hyperstructures and their Representations, Hadronic Press, Palm Harbor, USA, 1994. 